\title{
Distinct expression profile of HCMV encoded miRNAs in plasma from oral lichen planus patients
}

Meng Ding ${ }^{1,2+}$, Xiang Wang ${ }^{3 \dagger}$, Cheng Wang ${ }^{1,2+} \mathbb{D}$, Xiaoshuang Liu ${ }^{2}$, Ke Zen ${ }^{2}$, Wenmei Wang ${ }^{3 *}$, Chen-Yu Zhang ${ }^{2^{*}}$ and Chunni Zhang ${ }^{1,2^{*}}$

\begin{abstract}
Background: Oral lichen planus (OLP) is a T cell-mediated autoimmune disease. The aetiology and molecular mechanisms of OLP remain unclear. Human cytomegalovirus (HCMV) infection is a causal factor in the development of various diseases, but the clinical relevance of HCMV in OLP has not been thoroughly investigated.

Methods: In the present study, we firstly examined twenty-three HCMV-encoded microRNA (miRNA) expression profiles in plasma from training set that including 21 OLP patients and 18 healthy controls using RT-qPCR technology. Dysregulated miRNAs were subsequently confirmed in another larger cohort refereed as validation set consisting of 40 OLP patients and 33 healthy controls. HCMV DNA in peripheral blood leukocytes (PBLs) was also measured in an additional cohort of 13 OLP patients and 12 control subjects. Furthermore, bioinformatics analyses, luciferase reporter assay and western blotting were also performed to predict and verify the direct potential targets of HCMV-encoded miRNAs.

Results: The RT-qPCR results showed that the plasma levels of five HCMV-encoded miRNAs including hcmvmiR-UL112-3p, hcmv-miR-UL22a-5p, hemv-miR-UL148d, hcmv-miR-UL36-5p and homv-miR-UL59 were significantly increased in OLP patients in both training and validation sets. HCMV DNA in PBLs was also significantly higher in OLP patients than in control subjects. Additionally, by using a combination of luciferase reporter assay and western blotting, we demonstrated that cytomegalovirus UL16-binding protein 1, a molecule that mediates the killing of virusinfected cells by natural killer cells, is a direct target of hcmv-miR-UL59.
\end{abstract}

Conclusions: Our results demonstrate a distinct expression pattern of HCMV-encoded miRNAs in OLP patients, which may provide insight into the relationship between HCMV infection and OLP, and warrants additional study in the diagnosis and aetiology of OLP.

Keywords: Cytomegalovirus, MicroRNAs, Oral lichen planus, Cytomegalovirus UL16-binding protein 1, Plasma

\footnotetext{
*Correspondence: wenmei-wang@hotmail.com; cyzhang@nju.edu.cn; zchunni27@hotmail.com

${ }^{\dagger}$ Meng Ding, Xiang Wang and Cheng Wang contributed equally to this work

2 State Key Laboratory of Pharmaceutical Biotechnology, Jiangsu Engineering Research Center for MicroRNA Biology and Biotechnology, Advance Research Institute of Life Sciences, Nanjing University School of Life Sciences, Nanjing University, Nanjing 210046, China

${ }^{3}$ Department of Oral Medicine, Nanjing Stomat-ological Hospital, Medical School of Nanjing University, Nanjing 210000, China Full list of author information is available at the end of the article
} 


\section{Background}

Oral lichen planus (OLP) is a relatively common chronic inflammatory disease of the oral mucosa that affects $0.1-4.0 \%$ of the adult population, with middle-aged and aged females exhibiting higher incidences $[1,2]$. OLP affects oral mucous membranes with a variety of clinical presentations, including reticular, erosive, plaquelike, atrophic and ulcerative lesions [3], and the reticular and erosive type are most common clinically. Various therapeutic regimens have been designed to treat OLP. However, the highly relapsing nature of OLP is a great clinical challenge to improve the treatment result of the disease. Moreover, OLP is classified as a precancerous condition by the World Health Organization (WHO) [4]. Opinions on the pathogenesis of OLP vary, but it is currently accepted that OLP is a T-cell-mediated autoimmune disease [5]. However, the aetiology and molecular mechanisms underlying this autoimmune disease remain unclear.

MicroRNAs (miRNAs) are a subset of non-coding RNA molecules (21-23 nucleotides in length) that mediate post-transcriptional gene silencing [6]. MiRNAs are involved in various physiological and pathological processes, such as inflammatory and immune responses [7], in addition to their remarkable regulatory roles in the initiation and progression of human malignancies $[8,9]$. Mounting discoveries demonstrated that serum or plasma contain a large amount of stable miRNAs that are derived from the host itself and other species, such as viruses, that infect the host, and certain dysregulated miRNAs in circulation are involved in the pathological processes of human diseases [10-12].

Human cytomegalovirus (HCMV) is a member of the herpesviridae family,betaherpesvirinae subfamily that latently infects approximately $70-100 \%$ of the population worldwide for their lifetime [13]. HCMV infection is generally asymptomatic in immunocompetent individuals, but it is also implicated in cardiovascular disease, arthritis and aggressive brain tumours [14, 15]. Since the report in 2004 that virus express miRNAs [12], at least $26 \mathrm{HCMV}$-encoded mature miRNAs have been discovered [16]. Some HCMV-associated miRNAs are related to viral proliferation, latency and immunoevasion [17]. For example, hcmv-miR-UL112-3p is highly expressed in plasma of hypertensive patients and associated with an increased risk of hypertension [18]. HcmvmiR-UL112-3p targets IRF-1 and MICB, which are two key molecules in immunological, inflammatory and antiinfective responses, and IRF-1 and MICB repression by hcmv-miR-UL112-3p may be a unifying mechanism that evades the host response in the pathogenesis of hypertension [18]. A higher prevalence of homv-miR-UL112$3 p$ was also detected in patients with diabetes mellitus and glioblastoma multiforme [19]. Hcmv-miR-US4-1 also targets ERAP1b mRNA during viral infection, which resulted in the inhibition of cytotoxic $\mathrm{T}$ lymphocyte (CTL) immune responses [20]. Our previous study showed that serum homv-miR-US4-1 can serve as a novel biomarker for predicting the outcome of interferon $\alpha$ treatment in chronic hepatitis B patients [21].

The clinical relevance and significance of HCMV in OLP have not been thoroughly investigated. This study examined HCMV-encoded miRNA expression profile in the plasma from patients with OLP. We found that the plasma expression pattern of HCMV-encoded miRNAs in patients with OLP was different from normal controls, and 5 of 23 studied HCMV miRNAs were upregulated in OLP patients. HCMV DNA in peripheral blood leukocytes (PBLs) was also significantly higher in OLP patients than in control subjects. While the positive rate of anti-HCMV IgG and antiHCMV IgM were not different between OLP patients and normal control, which is consistent with the previous report [22]. Furthermore, we demonstrated that CMV UL16-binding protein 1 (ULBP1), which is a molecule that mediates the killing of virus-infected cells by natural killer (NK) cells, is a direct target of hcmvmiR-UL59. Together, our results demonstrate a set of upregulated HCMV-encoded miRNAs in OLP patients, which warrants additional study in the diagnosis and aetiology of OLP.

\section{Methods}

\section{Patients, sample collection and RNA isolation}

The present study enrolled 95 OLP patients, all of whom were newly diagnosed and have not yet been treated at Nanjing Stomatological Hospital. In addition, the recruitment of 63 subjects to the parallel control group was conducted in the Healthy Physical Examination Centre of Jinling Hospital. The health checkup included a detailed history, physical and ultrasonographic examinations and blood tests. The detailed clinical and grouped information can be seen in Table 1. All blood samples were collected in EDTA tubes using a standard operating procedure, and centrifuged and stored as previously described [23]. Total RNA was extracted from $100 \mu \mathrm{L}$ plasma using a 1-step phenol/chloroform purification protocol as previously described [24]. In brief, $100 \mu \mathrm{L}$ plasma was mixed with $300 \mu \mathrm{L}$ deionized water, $200 \mu \mathrm{L}$ acid phenol, and $200 \mu \mathrm{L}$ chloroform. The mixture was vortex-mixed vigorously and incubated at room temperature for $15 \mathrm{~min}$. After phase separation, the aqueous layer was mixed with 1.5 volumes of isopropyl alcohol and 0.1 volumes of $3 \mathrm{~mol} / \mathrm{L}$ sodium acetate $(\mathrm{pH} 5.3)$. This solution was stored at $-20^{\circ} \mathrm{C}$ for $1 \mathrm{~h}$. The RNA pellet was collected by centrifugation at $16,000 \mathrm{~g}$ for $20 \mathrm{~min}$ 
Table 1 Demographic and clinical features of OLP patients and healthy controls in the present study

\begin{tabular}{|c|c|c|c|c|c|c|c|c|c|c|}
\hline \multirow[t]{2}{*}{ Variable } & \multicolumn{3}{|l|}{ Training set } & \multicolumn{3}{|l|}{ Validation set } & \multicolumn{3}{|c|}{ HCMV titers set } & \multirow{2}{*}{$\begin{array}{l}\text { Exosome set } \\
\text { Cases }\end{array}$} \\
\hline & Cases & Controls & $P$ value & Cases & Controls & $P$ value & Cases & Controls & P value & \\
\hline Number & 21 & 18 & & 41 & 33 & & 13 & 12 & & 20 \\
\hline Age, years ${ }^{\mathrm{a}}$ & 46.48 (9.08) & $44.78(4.18)$ & $0.47^{b}$ & 46.59 (13.19) & $46.45(4.97)$ & $0.96^{\mathrm{b}}$ & $49.3(6.2)$ & $46.9(5.5)$ & $0.68^{b}$ & $45.6(6.3)$ \\
\hline Sex, n & & & $0.75^{c}$ & & & $0.84^{c}$ & & & $0.87^{c}$ & \\
\hline Male & $6(29 \%)$ & $6(33 \%)$ & & $13(32 \%)$ & $12(39 \%)$ & & $5(38 \%)$ & $5(41 \%)$ & & $7(35 \%)$ \\
\hline Female & $15(71 \%)$ & $12(67 \%)$ & & $28(68 \%)$ & $21(61 \%)$ & & $8(62 \%)$ & $7(59 \%)$ & & $13(65 \%)$ \\
\hline \multicolumn{11}{|l|}{ Type of OLP, n } \\
\hline Reticular OLP & $10(48 \%)$ & & & $21(51 \%)$ & & & $7(54 \%)$ & & & $10(50 \%)$ \\
\hline Erosive OLP & $11(52 \%)$ & & & $20(49 \%)$ & & & $6(46 \%)$ & & & $10(50 \%)$ \\
\hline Anti-HCMV IgG, n & & & $0.27^{c}$ & & & $0.11^{c}$ & & & $0.29^{c}$ & \\
\hline Positive & $21(100 \%)$ & $17(94 \%)$ & & 41 (100\%) & 31 (94\%) & & $13(100 \%)$ & 11 (92\%) & & $20(100 \%)$ \\
\hline Negative & $0(0 \%)$ & $1(6 \%)$ & & $0(0 \%)$ & $2(6 \%)$ & & $0(0 \%)$ & $1(8 \%)$ & & $0(0 \%)$ \\
\hline \multicolumn{11}{|l|}{ Anti-HCMV IgM, n } \\
\hline Positive & $0(0 \%)$ & $0(0 \%)$ & & $0(0 \%)$ & $0(0 \%)$ & & $0(0 \%)$ & $0(0 \%)$ & & $0(0 \%)$ \\
\hline Negative & $21(100 \%)$ & $18(100 \%)$ & & 41 (100\%) & $33(100 \%)$ & & $13(100 \%)$ & $12(100 \%)$ & & $20(100 \%)$ \\
\hline $\mathrm{HCV}, \mathrm{n}$ & & & $0.18^{c}$ & & & $0.04^{c}$ & & & $0.16^{c}$ & \\
\hline Positive & $2(9.5 \%)$ & $0(0 \%)$ & & $5(12.2 \%)$ & $0(0 \%)$ & & $2(15.4 \%)$ & $0(0 \%)$ & & $3(15 \%)$ \\
\hline Negative & 19 (90.5\%) & $18(100 \%)$ & & $36(87.8 \%)$ & $33(100 \%)$ & & $11(74.6 \%)$ & $12(100 \%)$ & & $17(85 \%)$ \\
\hline $\mathrm{EBV}, \mathrm{n}$ & & & & & & $0.2^{c}$ & & & & \\
\hline Positive & $0(0 \%)$ & $0(0 \%)$ & & $2(4.9 \%)$ & $0(0 \%)$ & & $0(0 \%)$ & $0(0 \%)$ & & $1(5 \%)$ \\
\hline Negative & $21(100 \%)$ & $18(100 \%)$ & & 39 (95.1\%) & $33(100 \%)$ & & 13 (100\%) & $12(100 \%)$ & & $19(95 \%)$ \\
\hline \multicolumn{11}{|l|}{$\mathrm{HIV}, \mathrm{n}$} \\
\hline Positive & $0(0 \%)$ & $0(0 \%)$ & & $0(0 \%)$ & $0(0 \%)$ & & $0(0 \%)$ & $0(0 \%)$ & & $0(0 \%)$ \\
\hline Negative & $21(100 \%)$ & 18 (100\%) & & 41 (100\%) & $33(100 \%)$ & & $13(100 \%)$ & $12(100 \%)$ & & 20 (100\%) \\
\hline
\end{tabular}

a Age data are presented as the mean \pm SD

b Student $t$ test

c Two-sided $x^{2}$ test

at $4{ }^{\circ} \mathrm{C}$. The resulting RNA pellet was washed once with $750 \mathrm{~mL} / \mathrm{L}$ ethanol and dried for $10 \mathrm{~min}$ at room temperature. The pellet was dissolved in $25 \mu \mathrm{L}$ of RNase-free water and stored at $-80{ }^{\circ} \mathrm{C}$ until further processed. For RNA isolation from cells, total RNA was extracted from the cultured cells using Trizol reagent (Invitrogen, MA, USA) according to the manufacturer's instructions.

\section{RT-qPCR analysis}

We performed a TaqMan probe-based RT-qPCR assay according to the manufacturer's instructions (Lightcycler ${ }^{\circledR} 480$ II, Roche) to investigate the differential expression of HCMV-encoded miRNAs between OLP patients and normal controls as described previously [24]. U6 small noncoding RNA (Applied Biosystems, Foster City, CA, USA) was used as a housekeeping gene to normalize the miRNA expression, and U6 expression levels were not significantly different between the two groups (Additional file 1: Figure S1). All reactions, including non-template controls, were performed in triplicate. The RT stem-loop primers and PCR primers (Applied Biosystems, Foster City, CA, USA) were highly specific for each target miRNA.

We assessed the detection limits of the RT-qPCR assay by conducting calibration curves developed with corresponding synthetic miRNA oligonucleotides (Additional file 1: Figure S2). All reactions were performed in triplicate. MiRNA expression in cells was also normalized to U6 small noncoding RNA. For mRNA quantification of ULBP1, total RNA was reverse transcribed into cDNA using oligo (dT). Real-time qPCR was performed using SYBR Green and normalized to GAPDH. The following PCR primers were used: ULBP1 forward: $\quad 5^{\prime}$-GTACTGGGAACAAATGCTGGAT-3'; ULBP1 reverse: 5'-AACTCTCCTCATCTGCCAGCT-3'; GAPDH forward, 5'-TGAAGCAGGCATCTGAGGG-3'; and GAPDH reverse, 5'-CGAAG GTGAAGAGTGGG AG- $3^{\prime}$. The relative content of miRNA and mRNA were calculated using the $2^{-\Delta \mathrm{Cq}}$ method and $2^{-\Delta \Delta \mathrm{Cq}}$ method, respectively. 


\section{HCMV titers}

We tested the copy numbers of HCMV in peripheral blood leukocytes (PBLs) by quantitative PCR in an additional 13 OLP patients and 12 healthy control subjects. DNA from PBLs (separated by Haoyang, Tianjin, China) was extracted according to the manufacturer's protocols of QIAamp DNA Mini kit (Qiagen, Hilden, Germany). We measured HCMV DNA using a TaqMan real-time PCR assay with the following HCMVspecific primers: HCMV DNA forward: 5'-CACGG TCCCGGTTTAGCA-3', HCMV DNA reverse: 5'-CG TAACGTGGACCTGACGTTT-3', FAM-labelled probe: 5'-FAM-TATCTGCCCGAGGATCGCGGTTACA-TA MRA-3'. Ten-fold diluted recombinant plasmid that contains the HCMV target sequence was used as a template for standard curve preparation (Additional file 1: Figure S3). Cq values were converted to absolute values from the standard curve. The 2-step thermocycling procedure consisted of 45 cycles of denaturation at $95{ }^{\circ} \mathrm{C}$ for $15 \mathrm{~s}$, annealing and extension at $60{ }^{\circ} \mathrm{C}$ for $60 \mathrm{~s}$. Results were expressed as copies per $1 \mathrm{~mL}$ blood.

\section{Determination of anti-HCMV IgG and IgM antibodies in plasma}

Enzyme-linked immunosorbent assay (ELISA) was performed to detect anti-HCMV IgG and IgM antibodies in plasma using a HCMV IgG/IgM kit (MEDSON, NJ, USA) according to the manufacturer's instructions. For the IgG-ELISA, a calibration curve, calibrated against the 1st WHO international standard, was used to quantitatively determine IgG antibody concentrations in each sample. For the IgM-ELISA, the test results were calculated using the optical density (OD) value at $450 \mathrm{~nm}$, and the cut-off value for positivity was $\mathrm{OD}>1.2$.

\section{Cell culture and luciferase reporter assays}

The human embryonic kidney cell line HEK293 was obtained from the Shanghai Institutes for Biological Sciences (Shanghai, China). The complete growth medium of HEK293 was Dulbecco's modified Eagle's medium (DMEM) (Gibco, NY, USA) and 10\% foetal bovine serum (Gibco, NY, USA). These cells were maintained at $37{ }^{\circ} \mathrm{C}$ under an atmosphere of $5 \% \mathrm{CO}_{2}-95 \%$ air. Luciferase reporter assays were performed to confirm that hcmvmiR-UL59 directly targeted the $3^{\prime}$ UTR of the ULBP1 gene. The $3^{\prime} U T R$ fragments from ULBP1 containing the predicted hcmv-miR-UL59 binding site were cloned into a pMIR-reporter plasmid (Ambion, Shanghai, China), and which was co-transfected into HEK293 cells with hcmv-miR-UL59 mimics by Lipofectamine 2000 (Thermo fisher, NY, USA). A $\beta$-galactosidase vector was co-transfected into HEK293 cells simultaneously as a transfection control. Cells were assayed using luciferase assay kits (Promega, Madison, WI, USA) at $24 \mathrm{~h}$ after transfection. The reported data represent three independent experiments.

\section{Western blot analysis}

Total cell lysates $(50 \mu \mathrm{g})$ from HEK293 cells were separated on $12 \%$ SDS-PAGE and transferred to polyvinylidenedifluoride (Bio-Rad, Hercules, CA, USA). The detailed protocol was described as following: briefly, after blocking in $20 \mathrm{mmol} / \mathrm{L}$ Tris- $\mathrm{HCl}, \mathrm{pH} \mathrm{7.6,} \mathrm{containing}$ $150 \mathrm{mmol} / \mathrm{L} \mathrm{NaCl}, 0.1 \%$ Tween 20, and 5\% (wt/vol) nonfat dry milk, the blots were incubated with the specific rabbit anti-human ULBP1 (Santa Cruz Biotechnology, CA, USA) and anti-GAPDH (Santa Cruz Biotechnology, CA, USA) overnight at $4{ }^{\circ} \mathrm{C}$. The blots were then incubated with horseradish peroxidase conjugated secondary antibody (Santa Cruz Biotechnology, CA, USA) for $1 \mathrm{~h}$ while shaking at room temperature. The autoradiographic intensity of each band was scanned and quantified using Image J software.

\section{Exosome RNA extraction and quantification}

Exosomes from the plasma samples of 20 OLP patients was isolated using the Total Exosome Isolation Kit (Invitrogen, MA, USA) according to the manufacturer's instructions. Exosomal RNA was extracted using a miRNeasy Mini Kit (Qiagen, Hilden, Germany) according to the manufacturer's instructions. Hcmv-miR-UL59 from exosomal RNA and plasma total RNA was quantified using RT-qPCR analysis. Ten-fold serial dilution of synthetic single-strand hcmv-miR-UL59 was used as a template for standard curve preparation (Additional file 1: Figure S2B). Cq values were converted to absolute values from the standard curve.

\section{Statistical analysis}

Statistical analyses were performed using SPSS software (version 19.0). Data are presented as the mean \pm SEM for miRNAs or mean \pm SD for other variables. A 2-sided Student's $t$ test was used to compare differences in variables between groups. ANOVA analysis was performed when compared the plasma levels of the 5 upregulated miRNAs in different types of OLP patients. Univariate and multivariate logistic regression analyses were performed to analyse the associations between plasma HCMV encoded miRNAs and OLP. A $P$ value of $<0.05$ was considered statistically significant.

\section{Results}

Expression profiles of HCMV-encoded miRNAs using RT-qPCR analysis

In the training set, $23 \mathrm{HCMV}$-encoded miRNAs were measured using a RT-qPCR assay in a cohort of individual 


\section{Training set: RT-qPCR analysis of twenty-three HCMV-encoded miRNAs in} individual plasma samples

Threshold: miRNAs with a fold change $>2.0$ and $P<0.05$

21 OLP cases from Nanjing Stomatological Hospital
18 healthy controls from Jinling Hospital

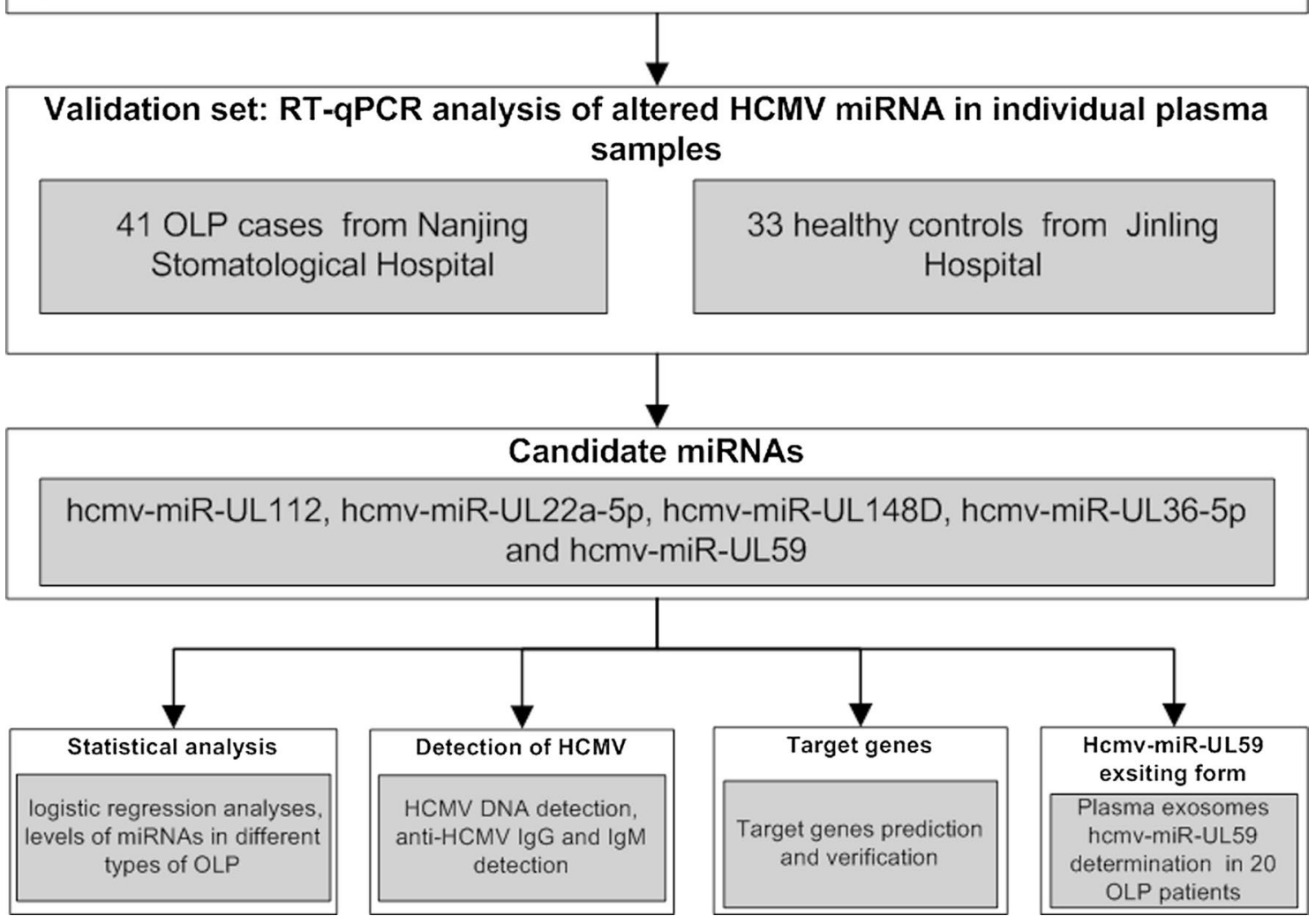

Fig. 1 Overview of the experimental design

plasma samples from 21 OLP patients and 18 healthy controls (Fig. 1). The RT-qPCR results showed that all of the examined $23 \mathrm{HCMV}$-encoded miRNAs were detectable in OLP patients and controls. Five of the 23 HCMV-encoded miRNAs, hcmv-miR-UL112-3p, hcmvmiR-UL22a-5p, hcmv-miR-UL148d, hcmv-miR-UL36$5 \mathrm{p}$ and hcmv-miR-UL59, were significantly upregulated in OLP samples compared with normal samples (fold change $>2, \mathrm{P}<0.05$ ) (Table 2). Our data suggest that the plasma expression pattern of HCMV-encoded miRNAs in OLP patients is different from healthy controls.

\section{Further confirmation of miRNAs}

Subsequently, the 5 selected miRNAs were further confirmed in an additional larger cohort of 40 OLP patients and 33 healthy controls (Fig. 1). Consequently, the alterations of the expression patterns of these 5 miRNAs in the OLP samples in this validation cohort were consistent with the results from the former cohort (Fig. 2; Additional file 1: Table S1). The differences in relative expression levels for the 5 altered miRNAs in all 61 OLP patients and 51 control individuals in the two cohorts are shown in Fig. 3a-e and Additional file 1: Table S2.

Plasma levels of the 5 identified miRNAs in different types of OLP

Oral lichen planus is categorized into several clinical forms. The most common categories of OLP are reticular OLP and erosive OLP. These two types of OLP are obviously distinguished from each other in clinical features, and the development of these diseases are also discrepant [25]. We evaluated changes in the plasma 
Table 2 Expression profile of HCMV-encoded miRNAs in OLP patients and normal controls in the training set

\begin{tabular}{|c|c|c|c|c|}
\hline HCMV encoded miRNAs & $\begin{array}{l}\text { OLP patients }(n=21) \\
\text { Mean } \pm \text { SEM }\end{array}$ & $\begin{array}{l}\text { Normal controls }(n=18) \\
\text { Mean } \pm \text { SEM }\end{array}$ & Fold change & $P$ value $^{\mathrm{a}}$ \\
\hline hcmv-miR-UL112-3p & $3.76 \pm 0.50$ & $0.52 \pm 0.06$ & 7.20 & $2.711 \times 10^{-7}$ \\
\hline homv-miR-UL112-5p & $0.29 \pm 0.03$ & $0.15 \pm 0.02$ & 1.89 & $2.532 \times 10^{-3}$ \\
\hline hemv-miR-UL22a-3p & $0.16 \pm 0.02$ & $0.13 \pm 0.02$ & 1.23 & 0.360 \\
\hline hemv-miR-UL22a-5p & $1.00 \pm 0.23$ & $0.37 \pm 0.08$ & 2.67 & 0.026 \\
\hline hcmv-miR-UL148d & $53.70 \pm 11.73$ & $19.94 \pm 4.94$ & 2.69 & 0.017 \\
\hline hcmv-miR-UL36-5p & $9.21 \pm 2.07$ & $1.72 \pm 0.42$ & 5.36 & 0.002 \\
\hline hcmv-miR-UL36-3p & $0.33 \pm 0.06$ & $0.22 \pm 0.04$ & 1.50 & 0.131 \\
\hline hemv-miR-UL59 & $3.98 \pm 0.61$ & $1.89 \pm 0.25$ & 2.11 & $2.034 \times 10^{-4}$ \\
\hline hemv-miR-UL69 & $0.02 \pm 0.20 \times 10^{-2}$ & $0.03 \pm 0.01$ & 0.76 & 0.308 \\
\hline hemv-miR-UL70-5 & $1.29 \pm 0.12$ & $1.54 \pm 0.17$ & 0.84 & 0.244 \\
\hline hcmv-miR-US22-3p & $0.53 \pm 0.06$ & $0.41 \pm 0.07$ & 1.28 & 0.192 \\
\hline hcmv-miR-US22-5p & $8.01 \pm 1.35$ & $6.07 \pm 0.72$ & 1.32 & 0.225 \\
\hline hemv-miR-US25-1 & $0.07 \pm 0.01$ & $0.05 \pm 0.01$ & 1.52 & 0.074 \\
\hline hemv-miR-US25-2-3p & $71.94 \pm 67.34$ & $2.57 \pm 0.40$ & 28.01 & 0.369 \\
\hline hemv-miR-US25-2-5p & $1.83 \pm 0.27$ & $1.23 \pm 0.12$ & 1.49 & 0.051 \\
\hline hcmv-miR-US29-3p & $0.05 \pm 0.01$ & $0.08 \pm 0.01$ & 0.63 & 0.055 \\
\hline hcmv-miR-US33-3p & $0.23 \pm 0.02$ & $0.20 \pm 0.02$ & 1.17 & 0.307 \\
\hline hcmv-miR-US33-5p & $0.72 \pm 0.08$ & $0.78 \pm 0.29$ & 0.92 & 0.800 \\
\hline hemv-miR-US4-3p & $54.00 \pm 46.89$ & $1.11 \pm 0.20$ & 48.53 & 0.304 \\
\hline hcmv-miR-US4-5p & $0.21 \pm 0.03$ & $0.31 \pm 0.04$ & 0.68 & 0.111 \\
\hline hemv-miR-US5-1 & $2.03 \pm 0.30$ & $1.77 \pm 0.40$ & 1.15 & 0.495 \\
\hline hcmv-miR-US5-2-3p & $0.04 \pm 0.01$ & $0.06 \pm 0.01$ & 0.72 & 0.040 \\
\hline hemv-miR-US5-2-5p & $3.60 \pm 0.56$ & $3.56 \pm 0.38$ & 1.01 & 0.800 \\
\hline
\end{tabular}

Data are presented as the mean \pm SEM

a Student $t$ test

levels of the aforementioned 5 miRNAs in OLP patients with different clinical types who enrolled in the training and validation cohorts $(n=61)$. As showed in Fig. $3 f-j$, hcmv-miR-UL112-3p and homv-miR-UL36-5p were upregulated in both reticular OLP and erosive OLP, whereas hcmv-miR-UL22a-5p and hcmv-miR-UL148d were only significantly upregulated in erosive OLP when compared with the normal controls. In addition, hcmvmiR-UL59 were only elevated in reticular OLP compared with the normal controls (Fig. 3j). However, no obvious difference in the levels of the 5 miRNAs was observed between the two different types of OLP (Fig. 3f-j; Additional file 1: Table S3).

\section{Elevated levels of HCMV encoded miRNAs in plasma are associated with the presence of OLP}

To evaluate the clinical usefulness of the five altered HCMV encoded miRNAs in plasma for OLP patients, we first performed a forward stepwise binary logistic regression using the status of OLP as the dependent variable. When using the control group as the reference category, three of the 5 selected miRNAs were independently correlated with OLP (Additional file 1: Table S4). The odds ratios (ORs) of these miRNAs for OLP were as follows: hcmv-miR-UL112-3p (OR $=6.044$, 95\% CI 1.659 22.024, $\mathrm{P}=0.006)$, hcmv-miR-UL36-5p $(\mathrm{OR}=5.565$, 95\% CI 1.520-20.374, $\mathrm{P}=0.010)$, homv-miR-UL59 $(\mathrm{OR}=5.565,95 \%$ CI $1.520-20.374, \mathrm{P}=0.010)$, hcmvmiR-UL22a-5p $(\mathrm{OR}=2.370,95 \%$ CI $0.595-9.447$, $\mathrm{P}=0.747)$ and hcmv-miR-UL148d $(\mathrm{OR}=3.077,95 \%$ CI $0.799-11.852, \mathrm{P}=0.501$ ). Furthermore, after adjusting for age, gender, CMV IgG, CMV IgM, HIV, HCV and EBV, hcmv-miR-UL112-3p still remained an independent association with OLP by multivariate logistic regression analyses using a dependent two-category variable (Additional file 1: Table S4).

\section{Detection of HCMV}

Because the levels of the 5 identified HCMV-encoded miRNAs were significantly upregulated in plasma from OLP patients, we investigated whether the latent infection of HCMV in OLP patients was different from that in the healthy controls. It has been reported that PBL-derived HCMV DNA has the higher sensitivity in 


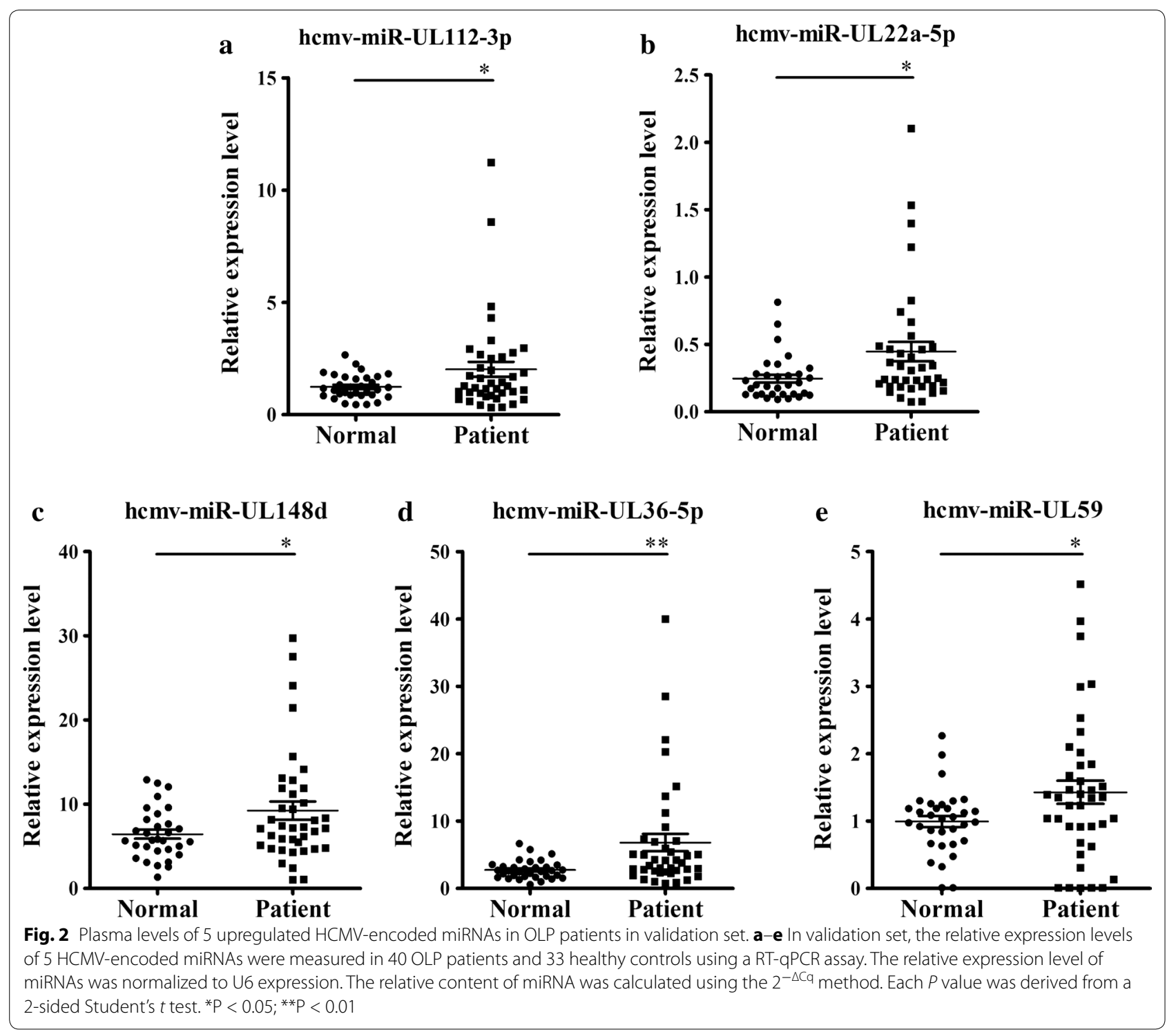

defining HCMV infection than plasma HCMV DNA in latency [26, 27]. Therefore, we detected HCMV DNA in PBLs of additional 13 OLP patients and 12 healthy controls using a quantitative PCR assay, and found that the HCMV DNA was significantly higher in OLP patients than in control subjects, with the concentrations were $(56.01-41,316) \pm 15,311$ copies/mL in OLP and (77.43-6065) \pm 2368 copies/mL in controls, respectively (Fig. 4a). Furthermore, we conducted ELISA analyses to measure the concentrations of anti-HCMV IgG and IgM in samples from both the training and validation sets. Our results revealed that almost all the subjects in both groups were anti-HCMV IgG positive and no significant differences were found in the plasma concentrations of anti-HCMV IgG between groups (Fig. 4b), neither the plasma positivity of anti-HCMV IgG and anti-HCMV IgM $(100.00 \%$ versus $93.65 \%, P=1.0 ; 0 \%$ versus $0 \%$, $P=1.0$, respectively). In addition, we also assessed the HCMV immediate early gene 1 (IE1) mRNA levels using RT-qPCR assay in the above patients and controls, nevertheless, none IE1 signal could be detected in both the blood of OLP patients and healthy controls.

\section{ULBP1 as a direct target of HCMV-miR-UL59}

To delineate the molecular mechanism underlying the role of dysregulated HCMV-encoded miRNAs in OLP, we used three algorithms, miR-Tar, miRanda and RNA hybrid, to locate HCMV miRNAs seed matches within human genes and HCMV genes to identify potential mRNA targets of these upregulated HCMV-encoded 

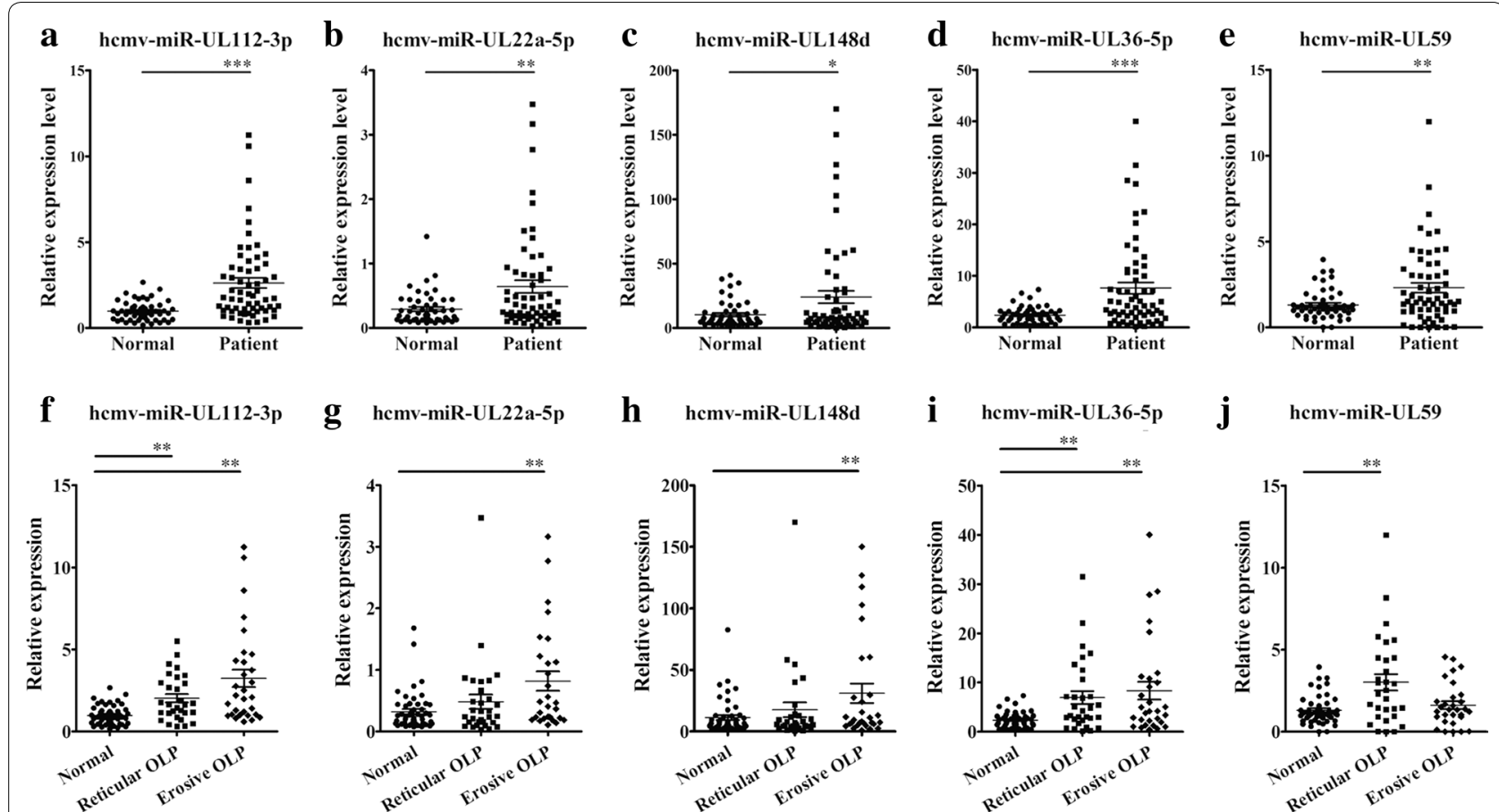

Fig. 3 Plasma levels of the 5 upregulated miRNAs in different types of OLP. a-e In training set and validation set, the relative expression levels of 5 HCMV-encoded miRNAs were measured in 61 OLP patients and 51 healthy controls using a RT-qPCR assay, each P-value was derived from a 2-sided Student's $t$ test; $\mathbf{f}-\mathbf{j}$ The relative expression levels of 5 upregulated HCMV-encoded miRNAs in 30 erosive OLP patients and 31 reticular OLP patients and 51 healthy controls were measured using RT-qPCR, each $P$ value was derived from ANOVA analysis. The relative content of miRNA was calculated using the $2^{-\Delta C a}$ method. The relative expression of miRNAs was normalized to U6 expression. ${ }^{*} P<0.05 ;{ }^{* *} \mathrm{P}<0.01$
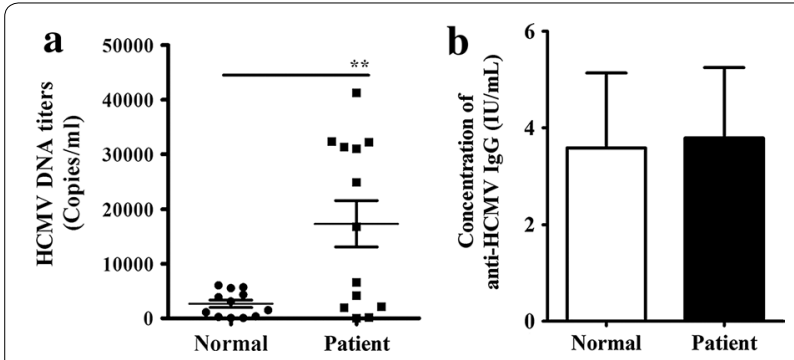

Fig. 4 Detection of HCMV and its relationship with HCMV-encoded miRNAs. a The HCMV DNA titers were calculated in PBLs of OLP patients and compared with healthy controls; $\mathbf{b}$ Comparison of the concentrations of anti-HCMV IgG in the OLP group $(n=61)$ versus the control group $(n=51) .{ }^{*} P<0.05$; ${ }^{* *} P<0.01$

miRNAs in OLP patients. Computational predictions indicated that some potential target genes of the 5 identified HCMV-encoded miRNAs are involved in immune regulation or viral replication or latency in HCMV or the regulation of apoptosis, tumours and cytokines in humans (Additional file 1: Table S5). Among these candidate targets, cytomegalovirus UL16-binding protein 1 (ULBP1), which is an NKG2D ligand, was predicted to be a potential target of hcmv-miR-UL59 (Additional file 1: Table S5). One predicted hybridization was observed between hcmv-miR-UL59 and the $3^{\prime}$-UTR of ULBP1, with perfect base-pairing between the seed region and the cognate target (Fig. 5a). The minimum free energy value of the hybridization between hcmv-miR-UL59 and ULBP1 is $-33.5 \mathrm{kcal} / \mathrm{mol}$, which is well within the range of genuine miRNA-target pairs (Fig. $5 \mathrm{a}$ ). The hcmv-miRUL59 binding sequence in the ULBP $3^{\prime}$-UTR was also highly conserved across primate species (Fig. 5a). The correlation between hcmv-miR-UL59 and ULBP1 was examined by evaluating ULBP1 expression in HEK293 cells after hcmv-miR-UL59 overexpression. The efficient overexpression of hcmv-miR-UL59 in HEK293 cells is shown in the Additional file 1: Figure S4. As expected, the transfection of HEK293 cells with hcmv-miR-UL59 mimics significantly decreased the ULBP1 protein levels (Fig. 5b), whereas the overexpression of hcmv-miRUL59 did not affect ULBP1 mRNA stability (Fig. 5c). Furthermore, we performed a luciferase reporter assay by transfecting HEK293 cells with a reporter vector that consisted of a luciferase gene containing the wild-type or mutated sequence of the $3^{\prime}$ UTR of ULBP1, including the hcmv-miR-UL59 binding site in its $3^{\prime} U T R$ region, 


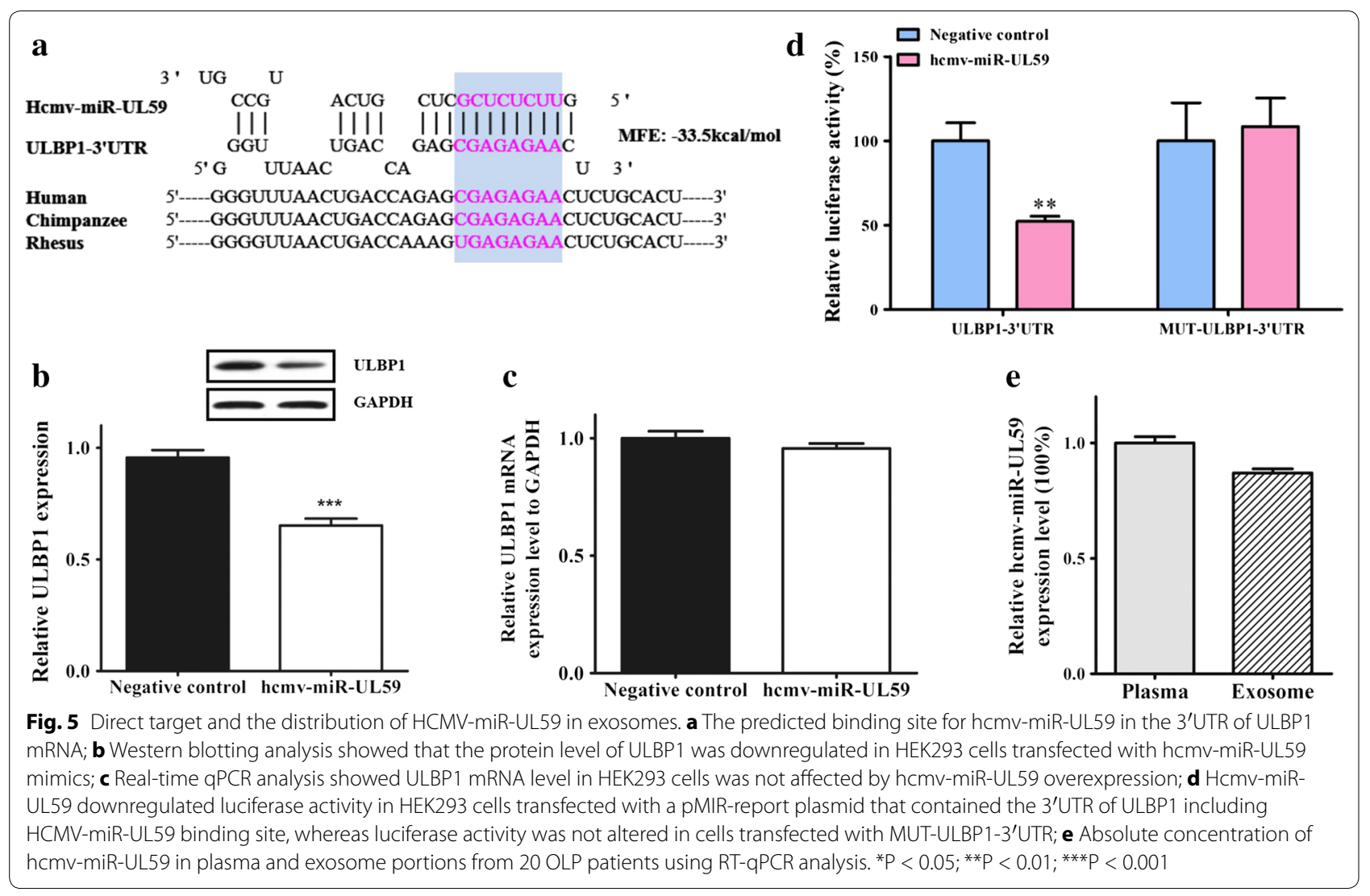

to confirm whether hcmv-miR-UL59 directly regulates ULBP1 (Fig. 5d). This experiment demonstrated that transfection with the WT-ULBP1-3'UTR plasmid inhibited approximately $50 \%$ of WT-ULBP1-3'UTR luciferase activity $(100 \%$ versus $52.27 \%, \mathrm{P}<0.01)$, but transfection with MUT-ULBP1-3'UTR did not significantly alter luciferase activity (100\% versus $108.45 \%, \mathrm{P}>0.05)$ (Fig. $5 \mathrm{~d}$ ). Taken together, these results confirm that ULBP1 is a direct and bona fide target of hcmv-miR-UL59.

\section{Plasma HCMV-miR-UL59 is primarily encapsulated in exosomes}

We suspected that plasma hcmv-miR-UL59 is primarily encapsulated in membrane-bound vesicles. To test our hypothesis, we determined the extent of plasma hcmvmiR-UL59 association with exosomes that were isolated from 20 OLP plasma samples. We observed that approximately $86 \%$ of plasma hcmv-miR-UL59 was contained in the exosome pellet (Fig. 5e). Furthermore, in order to confirm that hcmv-miR-UL59 was really encapsulated in exosomes, we firstly digested the isolated exosomes with RNase $\mathrm{H}$ for $1 \mathrm{~h}$ and then determined the levels of hcmv-miR-UL59 using qRT-PCR analysis, and found that the content of HCMV-miR-UL59 was not decreased after RNase treatment when compared with controls. Next, to test whether the exosomes protect the hcmv-miRUL59 from degradation by RNase $\mathrm{H}$, we disrupted the exosomes by adding $0.1 \%$ Triton $\mathrm{X}-100$ during the RNase $\mathrm{H}$ treatment. As shown in Additional file 1: Figure S5, the levels of hcmv-miR-UL59 were significantly decreased, which support our hypothesis that homv-miR-UL59 is associated with exosomes.

\section{Discussion}

Human cytomegalovirus infects most people worldwide, and it establishes a lifelong latency that must be suppressed by the immune system [28]. Cytomegalovirus is a clinically important pathogen that is associated with high levels of morbidity and mortality in immunocompromised individuals [29]. The majority of HCMV infections are asymptomatic, but these infections challenge our immune system by directly or indirectly inhibiting the function of some immune cells, particularly NK cells, and dysregulating cellular chemokines to their benefit $[14,30]$. HCMV may also be involved in the pathogenesis of diseases of unknown origin. In particular, Li et al. identified the association between HCMV infection and the development of essential hypertension through the dysregulated expression of hcmv-miR-UL112-3p [18]. Beside the above report, higher prevalence of 
hcmv-miR-UL112-3p was also detected in patients with diabetes mellitus and glioblastoma multiforme [19]. Pan et al. showed that serum HCMV-miR-US4-1 can serve as a novel biomarker for predicting the outcome of interferon $\alpha$ treatment in chronic hepatitis B patients [21]. Thus the availability of a highly sensitive test capable of detecting HCMV infection is of great significance. However, direct links between HCMV infection and OLP remain undefined. In this study, we examined the HCMV DNA in PBLs from our studied OLP patients, and the HCMV DNA in PBLs was significantly higher in OLP patients than in control subjects, while the positive rates of anti-HCMV IgG (past or current infection) and antiHCMV IgM (current or recent infection) were not different between OLP patients and controls. Interestingly, HCMV-encoded miRNAs were detectable in plasma samples from both OLP patients and controls. Notably, the plasma profile of HCMV-encoded miRNAs in OLP patients was different from normal controls, and 5 of 23 examined HCMV-encoded miRNAs were significantly upregulated in OLP patients. These results suggest that OLP patients have high loads of latent HCMV genomes and high levels of specific HCMV miRNAs compared to HCMV seropositive controls. In addition, our subsequent bioinformatics combined with luciferase analyzes and western blotting assays demonstrated that one of the HCMV encoded miRNA, homv-miR-UL59, could target ULBP-1, for which is a critical protein in immune elimination of HCMV. Thus, we suspected that HCMV may be involved in the pathogenesis of OLP, and those individuals with high latent loads of HCMV are perhaps more prone to OLP, nevertheless, further study are needed.

Notably, the majority of the 5 miRNAs identified in our study have been reported to be related to some HCMV infection diseases, for instance, hcmv-miR-UL22a-5p may be a potential predictor of clinical and virological endpoint in solid organ transplant patients [31], and increased hcmv-miR-UL112-3p was also reported to be related with glioblastoma, rheumatoid arthritis, diabetes mellitus essential hypertension as well as congenital infection $[18,19,32]$. All these results indicate that these HCMV encoded miRNAs may share similar physiological and pathological roles in HCMV infection related diseases as well as in OLP, eventhough much further study is needed to confirm this. On account of the unclear pathogenesis of OLP, our results may provide a clue to research the aetiology of OLP and insight into the role of circulating HCMV encoded miRNAs in HCMV infection in vivo.

Human cytomegalovirus has evolved a variety of mechanisms to evade host immune surveillance, which provides this virus with the capability of lifelong persistence in infected hosts in the form of a latent infection with the ability to reactivate when immune surveillance is compromised [33]. This study demonstrated that homvmiR-UL59 directly targeted UL16-binding protein 1 (ULBP1), which is a NKG2D ligand [33]. NKG2D is a key NK activating receptor that recognizes a family of stressinduced ligands, including MICA, MICB, and ULBP1-6 [34]. ULBP1 is induced by cellular stress and presented on the cell surface when cells are infected with HCMV, and ULBP1 interacts with NKG2D to mediate the killing of virus-infected cells by NK cells [14, 35]. Simultaneously, the HCMV glycoprotein UL16 binds to intracellular ULBP1 and inhibits its expression at the cell surface, which reduces the susceptibility of virus-infected cells to cytotoxic destruction by NK cells [36-38]. Hcmv-miRUL59 likely performs a congruent function as HCMV glycoprotein UL16. In addition, since ULBP1 is a critical protein in immune elimination of HCMV and also a novel potential target of HCMV-miR-UL-59, we speculated that intensive research on the functions of this newly regulatory relationship will help us to uncover the molecular mechanism of HCMV infection in future.

Exosomes are the major 'miRNA transporter' between cells for regional or long-distance communication [39, 40]. The vesicular structures contain membrane proteins similar to those of the donor cell and contain miRNA derived from their donor cell cytoplasm. They can be taken up and transfer their content to modulate cellular activities in recipient cells. Thus, the encapsulated miRNA have the ability to signal and regulate their target genes within the local microenvironment as well as at a distance [41]. The possible role of hcmv-miR-UL59 in the immunoevasion mechanism of HCMV suggests that hcmv-miR-UL59 is primarily entrapped in exosomes in plasma. We found that most hcmv-miR-UL59 (86\%) that was detectable in plasma samples from OLP patients was packed in exosomes during HCMV latency. Therefore, we speculated that the presence of homv-miR-UL59 and other upregulated HCMV-encoded miRNAs in the plasma of OLP patients might be a result of secreted exosomes from infected or inflammatory cells in which the virus reside. These cells may not secrete the HCMV but may secrete the HCMV encoded miRNAs into the circulation due to ongoing virus replication inside the cells in HCMV latency. These circulating miRNAs may be of some indicative value in HCMV infection.

The advantages of a miRNA-based regulation mechanism are multiple. These molecules are small, simpler to develop than a regulatory protein [42]. Furthermore, miRNAs are non-immunogenic molecules that allow viruses to regulate the expression of their host genome and establish an optimal environment while remaining undetected [43]. Unfortunately, we did not illuminate the specific underlying pathophysiological mechanisms between HCMV-encoded miRNAs and 
OLP. Hcmv-miR-UL112-3p targets MICB and IRF-1, which play critical roles in immunological, inflammatory, and anti-infection responses, and these proteins have a close relationship with essential hypertension [18, 44]. Hcmv-miR-UL148d downregulates IEX-1 expression, which contributes to the anti-apoptotic effects caused by ectopically expressed IEX-1 [45]. The chemokine RANTES (regulated on activation, expressed and secreted by normal T-cell) is a direct target of hcmv-miRUL148d, which provides a potential mean for immunosuppressive therapy against HCMV [29]. Despite these findings, the targets of HCMV-encoded miRNAs were not fully identified, and much data about their functions during infection and their relationship with human diseases are yet to be known.

\section{Conclusions}

In summary, we found that the expression profile of HCMV-encoded miRNAs in plasma samples from patients with OLP was different from that in normal controls, and 5 HCMV-encoded miRNAs were highly upregulated in OLP patients. Furthermore, we discovered that hcmv-miR-UL59 could target the cellular gene ULBP1, which is capable of mediating the immune elimination of HCMV-infected cells. A full understanding of the distinct express pattern of HCMV-encoded miRNAs and its relationship with OLP in vivo may lead to a broad clinical application.

\section{Additional file}

Additional file 1. Additional method. Quantification of miRNAs by TaqMan probe-based RT-qPCR. Figure S1. Cq Values of U6 in plasma samples from 61 OLP patients and 51 healthy controls. Figure S2. Standard curves of 5 HCMV encoded miRNAs. Figure S3. Standard curve of recombinant plasmid that contained the HCMV target sequence. Figure S4. The overexpression efficiency of hcmv-miR-UL59 in HEK293 cells. Figure S5. The relative expression levels of hcmv-miR-UL59 in plasma exosome, RNase $\mathrm{H}$ treated exosome and Triton/RNase $\mathrm{H}$ treated exosome. Table S1. The relative expression levels of HCMV-encoded miRNAs in OLP patients and normal controls in the validation set. Table S2. The relative expression levels of HCMV-encoded miRNAs in OLP patients and normal controls. Table S3. The relative expression levels of HCMV-encoded miRNAs in the two different types of OLP patients and normal controls. Table S4. Univariate and multivariate logistic regression analyses of plasma HCMV miRNAs for OLP. Table S5. Targets of HCMV-encoded miRNAs.

\section{Abbreviations}

OLP: oral lichen planus; miRNA: microRNA; HCMV: human cytomegalovirus; RT-qPCR: quantitative reverse transcription polymerase chain reaction; Cq: quantification cycle; ELISA: enzyme linked immunosorbent assay.

\section{Authors' contributions}

$C Z, C Y Z, W W$ and KZ conceived and designed the study. MD, CZ wrote the manuscript. $M D$ and $C W$ performed the experiments. MD and $X L$ performed the bioinformatics analysis and statistical analysis. WW, XW and CW contributed samples. All authors read and approved the final manuscript.

\begin{abstract}
Author details
${ }^{1}$ Department of Clinical Laboratory, Jinling Hospital, State Key Laboratory of Analytical Chemistry for Life Science, Advance Research Institute of Life Sciences, Nanjing University School of Life Sciences, Nanjing University, Nanjing 210002, China. ${ }^{2}$ State Key Laboratory of Pharmaceutical Biotechnology, Jiangsu Engineering Research Center for MicroRNA Biology and Biotechnology, Advance Research Institute of Life Sciences, Nanjing University School of Life Sciences, Nanjing University, Nanjing 210046, China. ${ }^{3}$ Department of Oral Medicine, Nanjing Stomat-ological Hospital, Medical School of Nanjing University, Nanjing 210000, China.
\end{abstract}

\section{Acknowledgements}

Not applicable.

\section{Competing interests}

The authors declare that they have no competing interests.

\section{Availability of data and materials}

All data generated or analysed during this study are included in this published article (and its additional files).

\section{Consent for publication}

OLP patients'samples and clinical information were collected and published under the guidelines for use of human samples that approved by ethics committee of Jinling Hospital and Nanjing Stomatological Hospital, with the written informed consent of all patients.

\section{Ethics approval and consent to participate}

All research and procedures involving human subjects (including the use of patient medical data and collection of blood) were reviewed and approved by the ethics committee of each participating hospital. Specifically, the methods in this study were carried out in accordance with the approved guidelines by Jinling Hospital and Nanjing Stomatological Hospital and all experimental protocols were approved by the ethics committees of the two hospitals. In addition, all patients and healthy controls provided written informed consent for participation.

\section{Funding}

This work was supported by the national basic research program of China (2014CB 542300) and the research special fund for public welfare industry of health of China (NO. 201302018) to C.Y.Z; the national natural science foundation of China (NSFC 81472021 and 81672102) to C.Z.; the national natural science foundation of China (NSFC 81401257) and the natural science foundation of Jiangsu province (BK20140730) to C.W.

\section{Publisher's Note}

Springer Nature remains neutral with regard to jurisdictional claims in published maps and institutional affiliations.

Received: 29 December 2016 Accepted: 23 May 2017

Published online: 07 June 2017

\section{References}

1. Arao TC, Guimaraes AL, de Paula AM, Gomes CC, Gomez RS. Increased miRNA-146a and miRNA-155 expressions in oral lichen planus. Arch Dermatol Res. 2012;304:371-5.

2. Danielsson K, Ebrahimi M, Wahlin YB, Nylander K, Boldrup L. Increased levels of COX-2 in oral lichen planus supports an autoimmune cause of the disease. J Eur Acad Dermatol Venereol. 2012;26:1415-9.

3. Lodi G, Scully C, Carrozzo M, Griffiths M, Sugerman PB, Thongprasom K. Current controversies in oral lichen planus: report of an international consensus meeting. Part 2. Clinical management and malignant transformation. Oral Surg Oral Med Oral Pathol Oral Radiol Endod. 2005;100:164-78.

4. Hu JY, Zhang J, Cui JL, Liang XY, Lu R, Du GF, Xu XY, Zhou G. Increasing CCL5/CCR5 on CD4+ T cells in peripheral blood of oral lichen planus. Cytokine. 2013;62:141-5. 
5. Liu GX, Sun JT, Yang MX, Qi XM, Shao QQ, Xie Q, Qu X, Wei FC, Sun SZ. OPN promotes survival of activated T cells by up-regulating CD44 in patients with oral lichen planus. Clin Immunol. 2011;138:291-8.

6. Bartel DP. MicroRNAs: genomics, biogenesis, mechanism, and function. Cell. 2004;116:281-97.

7. Huang CT, Oyang YJ, Huang HC, Juan HF. MicroRNA-mediated networks underlie immune response regulation in papillary thyroid carcinoma. Sci Rep. 2014;4:6495.

8. Lu J, Getz G, Miska EA, Alvarez-Saavedra E, Lamb J, Peck D, Sweet-Cordero A, Ebert BL, Mak RH, Ferrando AA, et al. MicroRNA expression profiles classify human cancers. Nature. 2005:435:834-8.

9. Baranwal S, Alahari SK. miRNA control of tumor cell invasion and metastasis. Int J Cancer. 2010;126:1283-90.

10. Chen X, Ba Y, Ma L, Cai X, Yin Y, Wang K, Guo J, Zhang Y, Chen J, Guo X, et al. Characterization of microRNAs in serum: a novel class of biomarkers for diagnosis of cancer and other diseases. Cell Res. 2008;18:997-1006.

11. Mitchell PS, Parkin RK, Kroh EM, Fritz BR, Wyman SK, Pogosova-Agadjanyan EL, Peterson A, Noteboom J, O'Briant KC, Allen A, et al. Circulating microRNAs as stable blood-based markers for cancer detection. Proc Natl Acad Sci USA. 2008;105:10513-8.

12. Soderberg-Naucler C, Nelson JY. Human cytomegalovirus latency and reactivation - a delicate balance between the virus and its host's immune system. Intervirology. 1999;42:314-21.

13. Pfeffer S, Zavolan M, Grasser FA, Chien M, Russo JJ, Ju J, John B, Enright AJ, Marks D, Sander C, Tuschl T. Identification of virus-encoded microRNAs. Science. 2004;304:734-6.

14. Baryawno N, Rahbar A, Wolmer-Solberg N, Taher C, Odeberg J, Darabi A, Khan Z, Sveinbjornsson B, FuskevAg OM, Segerstrom L, et al. Detection of human cytomegalovirus in medulloblastomas reveals a potential therapeutic target. J Clin Invest. 2011;121:4043-55.

15. Fielding CA, Aicheler R, Stanton RJ, Wang EC, Han S, Seirafian S, Davies J, McSharry BP, Weekes MP, Antrobus PR, et al. Two novel human cytomegalovirus NK cell evasion functions target MICA for lysosomal degradation. PLoS Pathog. 2014;10:e1004058.

16. Pavelin J, Reynolds N, Chiweshe S, Wu G, Tiribassi R, Grey F. Systematic microRNA analysis identifies ATP6VOC as an essential host factor for human cytomegalovirus replication. PLoS Pathog. 2013;9:e1003820

17. Grey F, Tirabassi R, Meyers H, Wu G, McWeeney S, Hook L, Nelson JA. A viral microRNA down-regulates multiple cell cycle genes through mRNA 5'UTRs. PLoS Pathog. 2010;6:e1000967.

18. Li S, Zhu J, Zhang W, Chen Y, Zhang K, Popescu LM, Ma X, Lau WB, Rong R, Yu X, et al. Signature microRNA expression profile of essential hypertension and its novel link to human cytomegalovirus infection. Circulation. 2011;124:175-84.

19. Mohammad AA, Rahbar A, Lui WO, Davoudi B, Catrina A, Stragliotto G, Mellbin L, Hamsten A, Ryden L, Yaiw KC, Soderberg-Naucler C. Detection of circulating hemv-miR-UL112-3p in patients with glioblastoma, rheumatoid arthritis, diabetes mellitus and healthy controls. PLOS ONE. 2014:9:e113740

20. Kim S, Lee S, Shin J, Kim Y, Evnouchidou I, Kim D, Kim YK, Kim YE, Ahn JH, Riddell SR, et al. Human cytomegalovirus microRNA miR-US4-1 inhibits CD8(+) T cell responses by targeting the aminopeptidase ERAP1. Nat Immunol. 2011;12:984-91.

21. Pan Y, Wang N, Zhou Z, Liang H, Pan C, Zhu D, Liu F, Zhang CY, Zhang Y, Zen K. Circulating human cytomegalovirus-encoded HCMV-miR-US4-1 as an indicator for predicting the efficacy of IFNalpha treatment in chronic hepatitis B patients. Sci Rep. 2016;6:23007.

22. Ghodratnama F, Wray D, Bagg J. Detection of serum antibodies against cytomegalovirus, varicella zoster virus and human herpesvirus 6 in patients with recurrent aphthous stomatitis. J Oral Pathol Med. 1999:28:12-5

23. Zhang C, Wang C, Chen X, Yang C, Li K, Wang J, Dai J, Hu Z, Zhou X, Chen $L$, et al. Expression profile of microRNAs in serum: a fingerprint for esophageal squamous cell carcinoma. Clin Chem. 2010;56:1871-9.

24. Wang C, Hu J, Lu M, Gu H, Zhou X, Chen X, Zen K, Zhang CY, Zhang T, Ge $J$, et al. A panel of five serum miRNAs as a potential diagnostic tool for early-stage renal cell carcinoma. Sci Rep. 2015;5:7610.

25. Sousa FA, Rosa LE. Oral lichen planus: clinical and histopathological considerations. Braz J Otorhinolaryngol. 2008;74:284-92.
26. Zhang S, Zhou YH, Li L, Hu Y. Monitoring human cytomegalovirus infection with nested PCR: comparison of positive rates in plasma and leukocytes and with quantitative PCR. Virol J. 2010;7:73.

27. Pellegrin I, Garrigue I, Ekouevi D, Couzi L, Merville P, Merel P, Chene G, Schrive MH, Trimoulet P, Lafon ME, Fleury H. New molecular assays to predict occurrence of cytomegalovirus disease in renal transplant recipients. J Infect Dis. 2000;182:36-42.

28. Nachmani D, Lankry D, Wolf DG, Mandelboim O. The human cytomegalovirus microRNA miR-UL112 acts synergistically with a cellular microRNA to escape immune elimination. Nat Immunol. 2010;11:806-13.

29. Kim Y, Lee S, Kim S, Kim D, Ahn JH, Ahn K. Human cytomegalovirus clinical strain-specific microRNA miR-UL148D targets the human chemokine RANTES during infection. PLoS Pathog. 2012;8:e1002577.

30. Hook LM, Grey F, Grabski R, Tirabassi R, Doyle T, Hancock M, Landais I, Jeng S, McWeeney S, Britt W, Nelson JA. Cytomegalovirus miRNAs target secretory pathway genes to facilitate formation of the virion assembly compartment and reduce cytokine secretion. Cell Host Microbe. 2014;15:363-73.

31. Lisboa LF, Egli A, O'Shea D, Asberg A, Hartmann A, Rollag H, Pang XL, Tyrrell DL, Kumar D, Humar A. Hcmv-miR-UL22A-5p: a biomarker in transplantation with broad impact on host gene expression and potential immunological implications. Am J Transplant. 2015;15:1893-902.

32. Kawano Y, Kawada J, Kamiya Y, Suzuki M, Torii Y, Kimura H, Ito Y. Analysis of circulating human and viral microRNAs in patients with congenital cytomegalovirus infection. J Perinatol. 2016;36:1101-5.

33. Lucin P, Mahmutefendic H, Blagojevic Zagorac G, llic Tomas M. Cytomegalovirus immune evasion by perturbation of endosomal trafficking. Cell Mol Immunol. 2015;12:154-69.

34. Seidel E, Le VT, Bar-On Y, Tsukerman P, Enk J, Yamin R, Stein N, Schmiedel D, Djian EO, Weisblum Y, Tirosh B. Dynamic co-evolution of host and pathogen: HCMV downregulates the prevalent allele MICA *008 to escape elimination by NK cells. Cell Reports. 2015;10:968-82.

35. Eagle RA, Trowsdale J. Promiscuity and the single receptor: NKG2D. Nat Rev Immunol. 2007;7:737-44.

36. Halenius A, Gerke C, Hengel H. Classical and non-classical MHC I molecule manipulation by human cytomegalovirus: so many targets-but how many arrows in the quiver? Cell Mol Immunol. 2015;12:139-53.

37. Dunn C, Chalupny NJ, Sutherland CL, Dosch S, Sivakumar PV, Johnson DC, Cosman D. Human cytomegalovirus glycoprotein UL16 causes intracellular sequestration of NKG2D ligands, protecting against natural killer cell cytotoxicity. J Exp Med. 2003;197:1427-39.

38. Cosman D, Mullberg J, Sutherland CL, Chin W, Armitage R, Fanslow W, Kubin M, Chalupny NJ. ULBPs, novel MHC class I-related molecules, bind to CMV glycoprotein UL16 and stimulate NK cytotoxicity through the NKG2D receptor. Immunity. 2001;14:123-33.

39. Gallo A, Tandon M, Alevizos I, Illei GG. The majority of microRNAs detectable in serum and saliva is concentrated in exosomes. PLoS ONE. 2012; $7:$ e30679.

40. Xu D, Tahara $\mathrm{H}$. The role of exosomes and microRNAs in senescence and aging. Adv Drug Deliv Rev. 2013;65:368-75.

41. Li L, Zhu D, Huang L, Zhang J, Bian Z, Chen X, Liu Y, Zhang CY, Zen K. Argonaute 2 complexes selectively protect the circulating microRNAs in cell-secreted microvesicles. PLoS ONE. 2012;7:e46957.

42. Cullen BR. Viruses and microRNAs. Nat Genet. 2006;38(Suppl):S25-30.

43. Kincaid RP, Sullivan CS. Virus-encoded microRNAs: an overview and a look to the future. PLoS Pathog. 2012;8:e1003018.

44. Miyamoto M, Fujita T, Kimura Y, Maruyama M, Harada H, Sudo Y, Miyata T, Taniguchi T. Regulated expression of a gene encoding a nuclear factor, IRF-1, that specifically binds to IFN-beta gene regulatory elements. Cell. 1988;54:903-13.

45. Wang YP, Qi Y, Huang YJ, Qi ML, Ma YP, He R, Ji YH, Sun ZR, Ruan Q. Identification of immediate early gene $\mathrm{X}-1$ as a cellular target gene of hcmv-mirUL148D. Int J Mol Med. 2013;31:959-66. 